\title{
Within-Session Practice Effects in Patients Referred for Suspected Dementia
}

\author{
Kevin Duff Gordon Chelune Kathryn Dennett \\ Department of Neurology, Center for Alzheimer's Care, Imaging and Research, University of Utah, \\ Salt Lake City, Utah, USA
}

\section{Key Words \\ Practice effects $\cdot$ Dementia $\cdot$ Cognition $\cdot$ Assessment}

\begin{abstract}
Background: Practice effects are improvements in cognitive test performance associated with repeated administrations of same or similar measures and are traditionally seen as error variance. However, there is growing evidence that practice effects provide clinically useful information. Methods: Within-session practice effects (WISPE) across 2 h were collected from 61 non-consecutive patients referred for suspected dementia and compared to the Mini Mental Status Examination (MMSE), a screening measure of dementia severity. Results: In all patients, WISPE on two cognitive measures were significantly correlated with MMSE, even after controlling for baseline cognitive scores (partial $r=0.47, p<0.001$; partial $r=0.26, p=0.046)$. In patients diagnosed with probable Alzheimer's disease, the trend was even stronger (partial $r=0.72, p<0.01$; partial $r=0.58, p$ $=0.046$ ). In both groups, lower WISPE were associated with lower MMSE scores (i.e. greater dementia severity), even after controlling for initial cognitive scores. Conclusion: If future research validates these findings with longitudinal studies, then WISPE may have important clinical applications in dementia evaluations.
\end{abstract}

Copyright $\odot 2012$ S. Karger AG, Basel
(C) 2012 S. Karger AG, Basel

$1420-8008 / 12 / 0334-0245 \$ 38.00 / 0$

Fax +41613061234

E-Mail karger@karger.ch

www.karger.com
Accessible online at: www.karger.com/dem

\section{Introduction}

Practice effects are improvements in cognitive test performance associated with multiple factors, including repeated administrations of the same or similar measures [1]. Traditionally, these improvements following readministration of a test are considered as systematic error variance that needs to be controlled. However, there is growing evidence that practice effects provide clinically useful information, especially in older adults with memory impairments. For example, practice effects seem to provide diagnostic information that separate intact elders from those with milder cognitive impairments. In these cases, intact individuals display the expected practice effects on retesting, whereas those with cognitive impairments show diminished practice effects [2-7]. Prognostically, practice effects across shorter retest intervals have been shown to predict cognitive outcomes across longer intervals in three different neuropsychiatric samples [8]. In a study focusing on amnestic mild cognitive impairment, practice effects across 1 week also predicted cognitive performance after 1 year, above and beyond baseline cognitive performance [9]. Practice effects may serve as a proxy of neural integrity, and when individuals stop benefitting from prior experiences (i.e. decreased practice effects), there functioning declines.

Practice effects have also been linked to treatment response. In one study, individuals with higher practice 
effects showed a better response to a memory training course than those with lower practice effects [10]. Learning potential is a construct similar to practice effects, in which within-session training on a cognitive test provides evidence of cognitive plasticity. Learning potential has been shown to predict training outcomes in older adults [11] and patients with schizophrenia [1214]. These studies seem to suggest that practice effects (or learning potential) can be used to identify those who might benefit from cognitive intervention, which could more appropriately utilize limited resources.

Although a number of studies have examined the prognostic value of practice effects in healthy elders and those with mild cognitive impairments, few have examined these clinical benefits of repeated testing among patients with dementia. The current study examined the relationship between within-session practice effects (WISPE) and current global functioning, a reasonable prognostic indicator in patients referred to a dementia clinic. We expected WISPE scores to be positively associated with current global functioning, with smaller practice effects correlating with lower global cognition, especially in amnestic conditions (e.g., amnestic mild cognitive impairment, Alzheimer's disease).

\section{Methods}

\section{Participants}

Sixty-one non-consecutive patients referred to a Cognitive Disorders Clinic for an evaluation for suspected dementia provided data for the current study. Their mean age was 73.3 years (standard deviation, SD 7.8) and the mean duration of their education was 14.7 years (2.8). The sample included slightly more females (56\%). Their premorbid intellect tended to be average (Test of Premorbid Functioning/Wechsler Test of Adult Reading $=42$ nd percentile, range $<1$ st-91st percentiles), but their current global cognition was borderline impaired [Mini Mental Status Examination (MMSE) $=25.5$ (SD 3.5), range 13-30]. On average, they reported only minimal depressive symptoms [30-item Geriatric Depression Scale 7.9 (SD 5.9)]. Following a thorough evaluation (described below), their diagnoses included: mild cognitive impairment (42\%), probable Alzheimer's disease (21\%), vascular cognitive impairment/ vascular dementia (10\%), frontotemporal dementia $(8 \%)$, depression $(5 \%)$, and other $(13 \%)$. The only inclusion criterion was that patients were able to complete the initial and repeated testing. No additional exclusion criteria were employed.

\section{Procedures}

All procedures, including the use of de-identified patient data, were approved by the local Institutional Review Board prior to study commencement. All patients referred to the de- mentia clinic underwent thorough evaluation by a board-certified neurologist with specialty training in dementia diagnosis and care. Each evaluation included: initial clinical interview with patient and collateral (if possible), mental status examination, review of systems, physical and neurological examination, laboratory work, magnetic resonance imaging, neuropsychological testing, positron emission tomography (if necessary to aid in differential diagnosis), and a second clinical visit to provide diagnostic impressions and treatment recommendations.

As part of a larger neuropsychological battery, all patients were administered the MMSE [15], a widely-used, 30-point measure of global cognition and dementia severity. The total raw score from the MMSE was used. Two other cognitive measures used as part of the neuropsychological evaluation were the Hopkins Verbal Learning Test Revised (HVLT-R) [16] and the Coding subtest of the Wechsler Intelligence Scale III/IV $[17,18]$. In the HVLT-R, participants are presented with 12 words to recall across three successive learning trials. The total number of words recalled across all three learning trials was used. In the Coding subtest, participants have $120 \mathrm{~s}$ to use a reference key to pair as many numeric digits with corresponding geometric figures. The number of correctly paired items was used. The HVLT-R and Coding subtest were performed twice during the neuropsychological evaluation, once in the beginning of the battery and once at the end (typically separated by approximately $2 \mathrm{~h}$ ). All neuropsychological testing was completed in a single session. Alternate test forms were not used for the HVLT-R or Coding subtest.

\section{Data Analysis}

To calculate WISPE, the scores from the initial administration of the HVLT-R and Coding subtest were subtracted from their respective repeated administration of the same measure (e.g., repeated HVLT-R - initial HVLT-R). The WISPE on the HVLT-R was correlated with the MMSE, after controlling for initial scores of the HVLT-R via partial correlations. Similarly, the WISPE on Coding subtest was correlated with the MMSE, after controlling for initial scores of coding via partial correlations. Given the limited number of analyses, $\alpha$ was set at 0.05 .

\section{Results}

Relevant cognitive test scores are presented in table 1. For the total sample, WISPE on the HVLT-R significantly correlated with the MMSE, after controlling for scores on the initial administration of the HVLT-R (partial $\mathrm{r}=$ $0.47, \mathrm{p}<0.001, \mathrm{~d}=1.06$ ). The Coding WISPE also significantly correlated with the MMSE, after controlling for initial Coding scores (partial $\mathrm{r}=0.26, \mathrm{p}=0.046, \mathrm{~d}=0.54$ ). When only those participants diagnosed with probable Alzheimer's disease were considered $(n=13)$, the HVLT-R WISPE continued to be significantly correlated with the MMSE (partial $\mathrm{r}=0.72, \mathrm{p}=0.009, \mathrm{~d}=2.06$ ), as did the Coding WISPE (partial $\mathrm{r}=0.58, \mathrm{p}=0.046, \mathrm{~d}$ 
Table 1. Cognitive test scores for all participants

\begin{tabular}{lcc}
\hline Measures & Mean \pm SD & Range \\
\hline MMSE & $25.5 \pm 3.5$ & $13-30$ \\
Initial HVLT-R & $17.5 \pm 6.4$ & $3-33$ \\
Repeated HVLT-R & $20.8 \pm 8.5$ & $1-36$ \\
WISPE HVLT-R & $3.4 \pm 4.4$ & -8 to 13 \\
Initial coding & $40.5 \pm 13.8$ & $8-77$ \\
Repeated coding & $43.4 \pm 15.8$ & $8-84$ \\
WISPE coding & $2.8 \pm 5.6$ & -23 to 16
\end{tabular}

$=1.44)$, even after their respective initial administration scores were partialled out. When only those diagnosed with mild cognitive impairment were considered $(\mathrm{n}=$ 26), there was a significant partial correlation between Coding WISPE and the MMSE (partial $\mathrm{r}=0.45, \mathrm{p}=$ $0.029, \mathrm{~d}=0.99$ ), but HVLT-R WISPE only trended in the expected direction (partial $\mathrm{r}=0.37, \mathrm{p}=0.073, \mathrm{~d}=0.80$ ). In all instances, lower WISPE were associated with lower MMSE scores, even after controlling for initial cognitive scores. The relationships between WISPE on the HVLT-R and MMSE for each group are presented in figure 1.

\section{Discussion}

Although practice effects have been previously examined in patients with mild cognitive impairment and dementia [19-22], few of these studies have considered the valuable clinical information that practice effects could provide. However, recent research suggests that practice effects may provide clinically useful information about diagnosis, prognosis and treatment response in older adults with memory impairments [3-5, 7, 9-11]. Results of the current study extend these prior findings by demonstrating that practice effects provide information about the severity of cognitive impairment and dementia in these older subjects. In our sample of patients referred for a dementia evaluation, WISPE on two cognitive measures were positively partially correlated with a measure of global cognition and dementia severity. Lower WISPE were associated with worse cognition/greater dementia severity. As we continue to follow these patients, it will be interesting to see if these baseline practice effects are also associated with future cognition (i.e. cognitive trajectories). However, it should be noted that the correlations between WISPE and MMSE were modest, with much additional variance unaccounted for.

WISPE in Patients Referred for Suspected Dementia

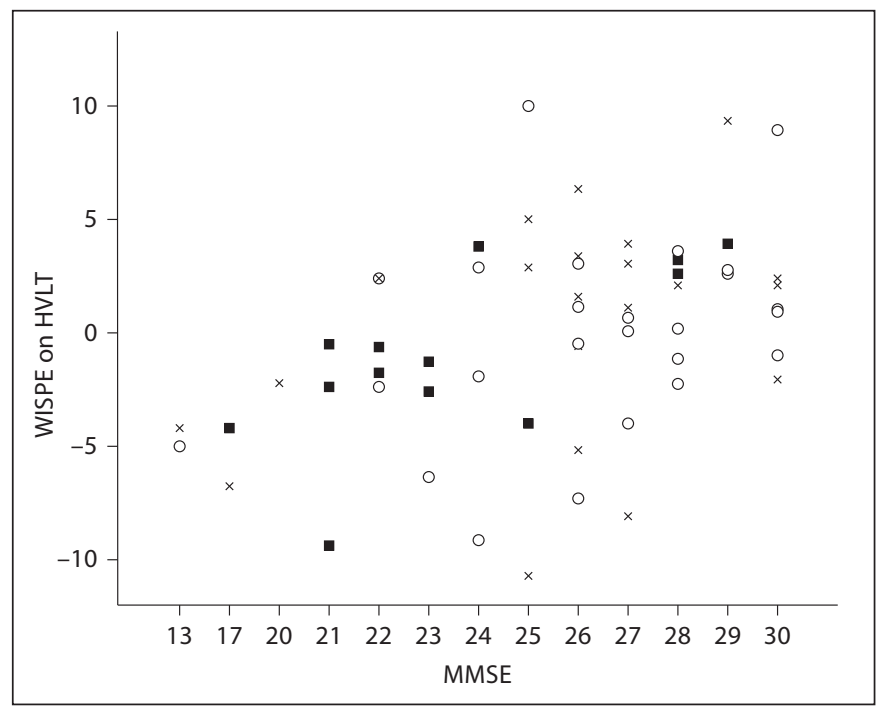

Fig. 1. Relationship between WISPE and MMSE. WISPE on HVLT $=$ WISPE on total recall of the HVLT corrected for initial score on this same measure; $\mathbf{\square}=$ probable Alzheimer's disease; $O=$ mild cognitive impairment; $\mathrm{x}=$ other.

Not only were the WISPE related to overall cognition in the entire sample, but subgroups of patients demonstrated relatively consistent results (fig. 1). For example, among those patients diagnosed with probable Alzheimer's disease, the relationship between WISPE and global cognition was stronger than for the entire sample. Less striking trends were observed in the subgroup of cases with mild cognitive impairment. Even in the remainder of the participants, which included a range of diagnoses (e.g., vascular dementia, frontotemporal dementia, depression), WISPE on the HVLT-R tended to be related to MMSE scores (partial $\mathrm{r}=0.33, \mathrm{p}=0.14, \mathrm{~d}=$ 0.69 ). These subgroup analyses suggest that practice effects provide valuable clinical information for a range of dementia-related conditions $[8,23]$.

Our subgroup analyses are also largely consistent with existing literature on practice effects across the range of dementia. In two studies by Cooper and colleagues [19, 20 ], $17-31 \%$ of patients with mild cognitive impairment or Alzheimer's disease displayed significant improvements on repeat testing (i.e. practice effects). Duff et al. [4] reported that nearly $50 \%$ of their participants with amnestic mild cognitive impairment displayed practice effects. As seen in figure 1, approximately $30 \%$ of patients diagnosed with probable Alzheimer's disease showed positive practice effects (i.e. improvement on repeated testing) and $46 \%$ of patients with mild cognitive impair- 
ment showed these practice effects. This accumulating evidence suggests that learning through practice is possible even in patients with severe cognitive disturbances, which could have implications for their diagnosis and treatment.

As reported in table 1, there is a surprising amount of variability in the WISPE scores in this dementia referral sample. For example, the standard deviations of the WISPE variables are quite large (even larger than the mean improvements on repeat testing). These large standard deviations, along with the partial correlations mentioned above, suggest that practice effects are not uniform across all patients, and they could serve as individual difference variables to enrich samples for clinical trials. For example, patients that do not demonstrate practice effects might be preferentially selected for clinical trials, as they may be progressing more quickly. Additionally, approximately one quarter of all cases showed worse scores on repeated testing within the same session (HVLT-R 20\%, Coding subtest 31\%). Conversely, in a study of community-dwelling and cognitively intact seniors [10], no participants showed within-session declines on a similar list learning test and only $8 \%$ showed declines on a similar coding task. These data continue to support the idea that an absence of practice effects is a poor indicator of current and future cognition.

Despite the growing body of literature to support the clinical value of practice effects, there are some limitations of the current study. First, the MMSE is a gross measure of cognition and dementia, and more sensitive methods could have been used. However, even with this bedside screening instrument, significant findings were observed. Second, data on non-consecutive patients were collected. Only those clinical cases that were thought to be able to complete the initial and repeated testing were funneled into this study. So it is possible that more impaired patients (e.g., MMSE <13) might have a different (or non-existent) relationship between practice effects and global cognition. Third, the current study examined the relationship between practice effects and current global cognition, when future cognition is probably more relevant. While we see this study as a first step in extracting useful clinical information from practice effects (e.g., dementia severity), we are continuing to follow these patients across time to more accurately determine the prognostic value of practice effects in dementia. Finally, although our interpretation of the findings support our hypothesis, other explanations may also be possible. For example, it is possible that practice effects reflect factors other than learning, such as cognitive fluctuations [24], examiner-examinee relationships, or patient fatigue [25].

\section{Acknowledgements}

The project described was supported by research grants from the National Institutes on Aging: K23 AG028417-01. The content is solely the responsibility of the authors and does not necessarily represent the official views of the National Institute on Aging or the National Institutes of Health.

\section{Conflict of Interest}

None.

\section{References}

$>_{1}$ Duff K, Beglinger LJ, Schoenberg MR, Patton DE, Mold J, Scott JG, Adams RL: Testretest stability and practice effects of the RBANS in a community dwelling elderly sample. J Clin Exp Neuropsychol 2005;27: 565-575.

2 Darby D, Maruff P, Collie A, McStephen M: Mild cognitive impairment can be detected by multiple assessments in a single day. Neurology 2002;59:1042-1046.

-3 Suchy Y, Kraybill ML, Franchow E: Practice effect and beyond: reaction to novelty as an independent predictor of cognitive decline among older adults. J Int Neuropsychol Soc 2011;17:101-111.
Duff K, Beglinger L, Van Der Heiden S, Moser D, Arndt S, Schultz S, Paulsen J: Shortterm practice effects in amnestic mild cognitive impairment: implications for diagnosis and treatment. Int Psychogeriatr 2008;20: 986-999.

-5 Fernandez-Ballesteros R, Zamarron MD, Tarraga L: Learning potential: a new method for assessing cognitive impairment. Int Psychogeriatr 2005;17:119-128.

-6 Calero MD, Navarro E: Relationship between plasticity, mild cognitive impairment and cognitive decline. Arch Clin Neuropsychol 2004;19:653-660.
Burkhart CS, Birkner-Binder D, Gagneux A, Berres M, Strebel SP, Monsch AU, Steiner LA: Evaluation of a summary score of cognitive performance for use in trials in perioperative and critical care. Dement Geriatr Cogn Disord 2011;31:451-459.

-8 Duff K, Beglinger L, Schultz S, Moser D, McCaffrey R, Haase R, Westervelt H, Langbehn $D$, Paulsen J: Practice effects in the prediction of long-term cognitive outcome in three patient samples: a novel prognostic index. Arch Clin Neuropsychol 2007;22:15-24.

\9 Duff K, Lyketsos CG, Beglinger LJ, Chelune G, Moser DJ, Arndt S, Schultz SK, Paulsen JS, Petersen RC, McCaffrey RJ: Practice effects predict cognitive outcome in amnestic mild cognitive impairment. Am J Geriatr Psychiatry 2011;19:932-939. 
10 Duff K, Beglinger LJ, Moser DJ, Schultz SK, Paulsen JS: Practice effects and outcome of cognitive training: preliminary evidence from a memory training course. Am J Geriatr Psychiatry 2010;18:91.

11 Calero MD, Navarro E: Cognitive plasticityas a modulating variable on the effects of memory training in elderly persons. Arch Clin Neuropsychol 2007;22:63-72.

12 Watzke S, Brieger P, Kuss O, Schoettke H, Wiedl KH: A longitudinal study of learning potential and rehabilitation outcome in schizophrenia. Psychiatr Serv 2008;59: 248-255.

$>13$ Fiszdon JM, McClough JF, Silverstein SM, Bell MD, Jaramillo JR, Smith TE: Learning potential as a predictor of readiness for psychosocial rehabilitation in schizophrenia. Psychiatry Res 2006;143:159-166.

- 14 Sergi MJ, Kern RS, Mintz J, Green MF: Learning potential and the prediction of work skill acquisition in schizophrenia. Schizophr Bull 2005;31:67-72.

-15 Folstein MF, Folstein SE, McHugh PR: 'Mini-mental state'. A practical method for grading the cognitive state of patients for the clinician. J Psychiatr Res 1975;12:189-198.
16 Brandt J, Benedict RHB: Hopkins Verbal Learning Test-Revised. Odessa, Psychological Assessment Resources, 1997.

17 Wechsler D: Wechsler Adult Intelligence Scale - Third Edition. San Antonio, The Psychological Corporation, 1997.

18 Wechsler D: Wechsler Adult Intelligence Scale - Fourth Edition. San Antonio, The Psychological Corporation, 2007.

19 Cooper DB, Epker M, Lacritz L, Weine M, Rosenberg RN, Honig L, Cullum CM: Effects of practice on category fluency in Alzheimer's disease. Clin Neuropsychol 2001;15: 125-128.

20 Cooper DB, Lacritz LH, Weiner MF, Rosenberg RN, Cullum CM: Category fluency in mild cognitive impairment: reduced effect of practice in test-retest conditions. Alzheimer Dis Assoc Disord 2004;18:120-122.

21 Yan JH, Dick MB: Practice effects on motor control in healthy seniors and patients with mild cognitive impairment and Alzheimer's disease. Neuropsychol Dev Cogn B Aging Neuropsychol Cogn 2006;13:385-410.
22 Zehnder AE, Blasi S, Berres M, Spiegel R, Monsch AU: Lack of practice effects on neuropsychological tests as early cognitive markers of Alzheimer disease? Am J Alzheimers Dis Other Demen 2007;22: 416-426.

23 Stein J, Luppa M, Maier W, Tebarth F, Heser K, Scherer M, Zimmermann T, Eisele M, Bickel H, Mosch E, Weyerer S, Werle J, Pentzek M, Fuchs A, Wiese B, Prokein J, Konig HH, Leicht H, Riedel-Heller SG: The assessment of changes in cognitive functioning in the elderly: age- and education-specific reliable change indices for the SIDAM. Dement Geriatr Cogn Disord 2012;33: 73-83.

24 Rongve A, Bronnick K, Ballard C, Aarsland $\mathrm{D}$ : Core and suggestive symptoms of dementia with lewy bodies cluster in persons with mild dementia. Dement Geriatr Cogn Disord 2010;29:317-324.

25 Buchman AS, Schneider JA, Leurgans S, Bennett DA: Physical frailty in older persons is associated with Alzheimer disease pathology. Neurology 2008;71:499-504. 